\title{
Eustachian tube function test as a predictor of middle ear barotrauma
}

\author{
Konstantin Petrov Georgiev ${ }^{1}$, Nikola Georgiev Shopov' ${ }^{\circledR}$ \\ ${ }^{1}$ MBAT Varna MMA Bulgaria, Varna, Bulgaria \\ ${ }^{2}$ Department of Aviation and Maritime Medicine, Military Medical Academy Sofia, Bulgaria
}

\begin{abstract}
Background: Most cases of middle ear barotraumas in divers are due to impassability of the Eustachian tube, and typically occur during diving or during compression and decompression in a hyperbaric chamber. The aim of our study is to compare the results of tympanometry and Valsalva part of Eustachian tube function test (ETF-test) with the ability of divers to compensate for the change in ambient pressure in a hyperbaric chamber and to evaluate the tests as predictors of middle ear barotraumas.

Materials and methods: The study included 35 professional divers undergoing annual medical examination. Using tympanometer we measured the intratympanic pressure at rest, and after the manoeuvre of Valsalva. Then all subjects underwent a barofunction test (BFT) to assess their diving fitness and the passability of the Eustachian tubes. In a typical BFT divers are compressing and decompressing to a pressure of 2.2 ATA for $1 \mathrm{~min}$ in a hyperbaric chamber. Based on results from previous studies we are using a 20 daPa cut-off point on the ETF test to predict Eustachian tube passability and a successful BFT.

Results: In the current study 24 divers received ETF test results higher than 20 daPa; 3 divers had ETF test values lower than $20 \mathrm{daPa}$ in both ears, but none of them displayed difficulties in the BFT; 8 divers had ETF values lower than 20 daPa in one ear and higher than 20 daPa in the other; 7 divers of the last group displayed difficulties with the BFT in the ear with poor ETF result.

Conclusions: We consider that the ETF test can be used to assess diving fitness as a screening method before performing a BFT, as values above 20 daPa guarantee Eustachian tube passability sufficient for diving activities. Values of $20 \mathrm{daPa}$ and less are not a definite predictor for the barofunction results. The results of the ETF test can also be used in the usual work of an otorhinolaryngologist to evaluate Eustachian function in cases of unilateral disease of middle ear.
\end{abstract}

(Int Marit Health 2020; 71, 3: 195-200)

Key words: Eustachian tube function test, Eustachian tube passability, barofunction test, middle ear barotrauma

\section{INTRODUCTION}

There are many tests for evaluation of Eustachian tube passability, such as Bluestone Nine-Step Test, Tubo-tympano-aerodynamic-graphy (TTAG), Tubomanometry, Sonotubometry, Continuous impedance, etc. Many publications evaluate instrumental findings in patients with barotrauma of the middle ear. However, studies of methods for assessing the risk of possible barotrauma are rare.

In XVIII century Valsalva proposed a simple test to evaluate the Eustachian tube passability. The Valsalva ma- noeuvre is a forceful exhalation against a closed airway. It is usually performed with the nostrils and mouth held shut, while blowing out air. This manoeuvre results in an increased nasopharyngeal pressure and opening of the Eustachian tubes [1]. It leads to an increase in intratympanic pressure and is commonly used in diving to equalise middle ear pressure during submersion. In XIX century Toynbee proposed another technique. The Toynbee manoeuvre is performed by pinching the nostrils shut while swallowing. The muscles in the back of the throat pull open the Eustachian tube and 


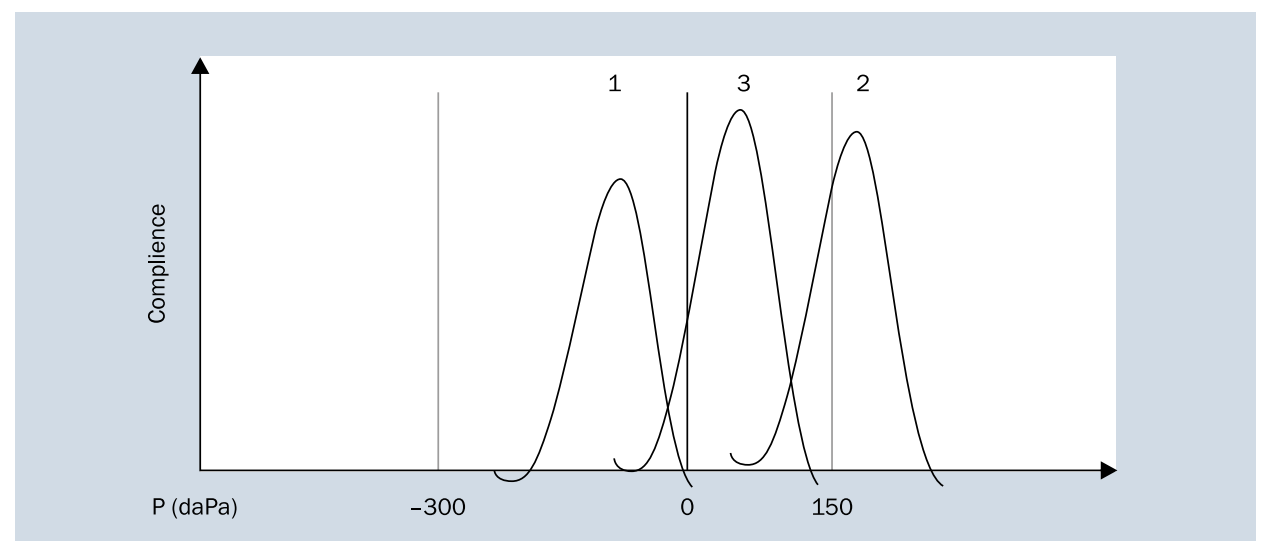

Figure 1. Normal Eustachian tube function test recorded by a tympanometer; 1 - intratympanic pressure during rest in daPa; 2 - pressure after the performing the manoeuvre of Valsalva; 3 - pressure after deep sniffing or after the Toynbee manoeuvre

allow air to equalise if a pressure gradient is present [2]. With the discovery of tympanometry, (Metz, 1948) it became possible to document these tests in charts and graphs.

Eustachian tube function (ETF) test is a test that examines the passability of the Eustachian tubes using a tympanometer. Figure 1 graphically presents the results of an ETF test. It consists of measuring the intratympanic pressure during rest, after performing the manoeuvre of Valsalva, and after deep sniffing or the Toynbee manoeuvre [3, 4]. The further away from curve 2 is from curves 1 and 3 , the better is the Eustachian tube passability. Tympanometry and ETF test can be performed even in a hyperbaric chamber [5].

Barofunction test (BFT) measures the ability of the body to react to fluctuations of the surrounding gas pressure. By design it is performed in a hyperbaric chamber. Usually divers perform manoeuvres of Valsalva and Toynbee to equalise the changes of surrounding gas pressure. In different countries the pressure in the hyperbaric chamber is increased from 1.0 up to 2.0-2.8 ATA (10-18 m water depth) for 40 seconds to a minute. In Bulgaria the protocol is 2.2 ATA (12 $\mathrm{m}$ water depth) for 1 minute. Barofunction test is used in the assessment of the suitability of diving training candidates or in the periodic examinations for diving fitness of professional divers. Since it is performed in a hyperbaric chamber with actual rapid change of the ambient pressure, divers occasionally suffer from barotrauma of the middle ear even during a barofunction test.

Additionally, non-divers are often treated in hyperbaric chambers with pressure changes of a much lower rate - 0.3 ATA/min. Occasionally, even in these soft regimes, patients suffer from barotrauma of paranasal sinuses and of the middle ear. Eustachian tube dysfunction and incorrect execution of the manoeuvres are the most important causes of barotraumas of the middle ear. This is why it is essential to find a quick and efficient method to screen out patients and divers with possible Eustachian tube dysfunction, so that middle ear barotraumas are prevented.
The purpose of the current study is to evaluate the ETF test as a predictor of middle ear barotraumas in divers. Based on previous studies we have estimated that ETF test values above 20 daPa guarantee Eustachian tube passability sufficient for diving activities [6]. Our hypothesis states that an ETF test value above that cut-off point (20 daPa) would mean that the diver will not suffer barotraumas during the BFT. This is how the 20 daPa cut-off point could be successfully used to screen for patients/ divers with possible Eustachian tube dysfunction.

\section{MATERIALS AND METHODS}

From November 2019 until January 2020 we performed annual examinations of 35 professional, highly motivated and qualified divers with different diving experience. With all subjects first we measured intratympanic pressure at rest, then after the manoeuvre of Valsalva, with MAICO MI-24 tympanometer. We used only the manoeuvre of Valsalva part of the ETF test, because most of the difficulties in the BFT appear at times of compression.

We compared the results of the ETF test with the results of a following barofunction test in "PDK-2" - hyperbaric chamber. We accepted that the ability to successfully tolerate compression up to 2.2 ATA for 1 minute is the criterion for normal passability of the Eustachian tube for divers.

All data were processed with MS Office Excel and IBM SPSS products.

We used a receiver operating characteristic (ROC) analysis to check the accuracy, sensitivity and specificity of the ETF test. ROC analysis is usually used to assess the diagnostic power of a binary classifier system, comparing it to a "gold standard". In this case the gold standard is the success in a BFT as described above. The graphical representation of this analysis is a ROC curve. It illustrates the true positive rate against the false positive rate at various threshold settings. The true-positive rate is also known as sensitivity, 
Table 1. Descriptive statistics of the divers sample

\begin{tabular}{llllll}
\hline Descriptive statistics & N & Minimum & Maximum & Mean & Standard deviation \\
\hline Pressure rest & 70 & -180.00 & 79.00 & -22.7571 & 31.41699 \\
ETF result & 70 & 8.00 & 226.00 & 74.6571 & 59.17792 \\
Difficulties & 70 & 0.00 & 1.00 & 0.1000 & 0.30217 \\
Valid N (listwise) & 70 & & & &
\end{tabular}

In the column $\mathrm{N}$ are included the number of ears tested, not subjects; ETF - Eustachian tube function

Table 2. Descriptive statistics of the subgroup of divers with unilateral difficulties

\begin{tabular}{llllll}
\hline Descriptive statistics & N & Minimum & Maximum & Mean & Standard deviation \\
\hline Pressure rest & 16 & -50 & 79 & -15.31 & 29.678 \\
ETF & 16 & 8 & 210 & 45.63 & 54.993 \\
Difficulties & 16 & 0 & 1 & 0.44 & 0.512 \\
Valid N (listwise) & 16 & & &
\end{tabular}

In the column $\mathrm{N}$ are included the number of ears tested, not subjects; ETF - Eustachian tube function

or probability of detection. The false-positive rate is also known as specificity or the probability of false alarm [7].

\section{ETHICAL CONSIDERATIONS}

The research is conducted in compliance with the ethical considerations and all principles for medical research involving human subjects according to the WMA Declaration of Helsinki.

\section{RESULTS}

In our sample 24 divers (68.6\% of the sample) received ETF-test results higher than 20 daPa. None of them displayed difficulties in the barofunction test. Three divers ( $8.6 \%$ of the sample) had ETF-test results lower than $20 \mathrm{daPa}$ (8-18 daPa) in both ears, but none of them displayed difficulties in the barofunction test. Eight divers $122.8 \%$ of all tested subjects) received ETF test results lower than $20 \mathrm{daPa}$ in one ear and higher than $20 \mathrm{daPa}$ in the other. Seven of them (20\% of all tested subjects and $87.5 \%$ of the subjects with unilateral poor ETF test results) had difficulties with the BFT in the ear with poor ETF result. One diver had ETF test result of 18 daPa - close to the critical value, but had no difficulties with the barofunction test.

We discovered subjects with large differences of normal intratympanic pressure in rest without any complaints or difficulties in barofunction (minimal pressure was -180 daPa, maximal pressure was $79 \mathrm{daPa}$, average pressure was -22.7571 daPa, standard deviation 31.417; Table 1).

In the subgroup of divers with unilateral difficulties we can assume that the failure on the BFT is due to Eustachian tube impassability, since the difficulties occur in one ear only. This means that the diver can apply a proper tech-
Table 3. Correlation between the intratympanic pressure at rest and the barofunction test results for all divers

\begin{tabular}{llll}
\hline \multicolumn{2}{l}{ Correlations } & Pressure rest & Difficulties \\
\hline Pressure & Pearson correlation & 1 & 0.141 \\
rest & Sig. (2-tailed) & & 0.245 \\
& N & 70 & 70
\end{tabular}

Table 4. Correlation between the intratympanic pressure at rest and the barofunction test results for divers with unilateral difficulties

\begin{tabular}{llll}
\hline \multicolumn{2}{l}{ Correlations } & Pressure rest & Difficulties \\
\hline Pressure & Pearson correlation & 1 & 0.176 \\
rest & Sig. (2-tailed) & & 0.514 \\
& $\mathrm{~N}$ & 16 & 16
\end{tabular}

nique but there is an objective cause for the failure on the BFT. This is why we analysed the results of this subgroup separately (Table 2).

We did not discover any significant correlation between intratympanic pressure at rest and difficulties in barofunction ( $r=0.141, p=0.245$ as shown in Table 2). These results correspond to the results from previous studies (Table 3) [6, 8].

Even in the subgroup of divers with unilateral difficulties we found no significant correlation between the intratympanic pressure at rest and the result from the barofunction test $(r=0.176, p=0.514)$. This result shows that the single value of intratympanic pressure is not related to and cannot provide reliable information about the Eustachian tube function (Table 4). 


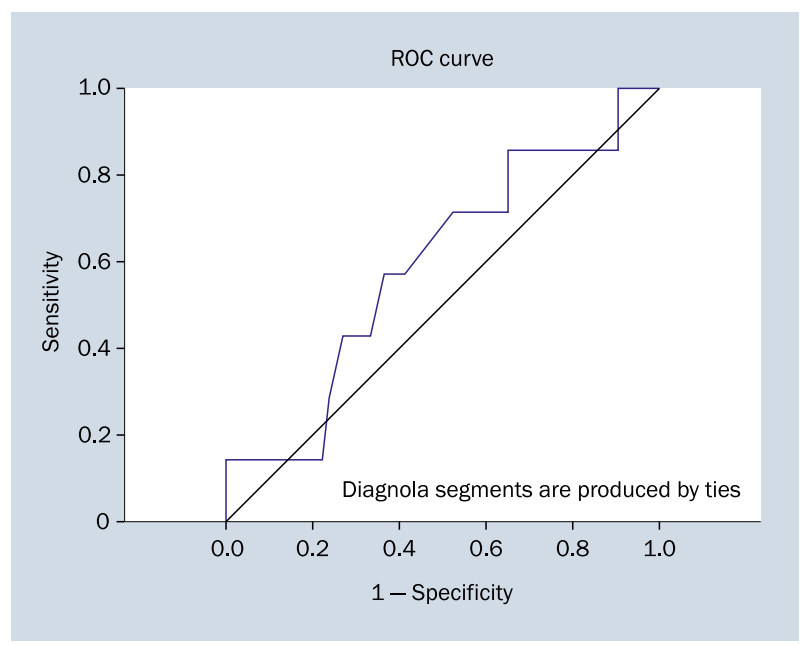

Figure 2. Receiver operating characteristic (ROC) analysis of intratympanic pressure in rest as predictor of barofunction result; area under the curve $=0.592$, standard error 0.112

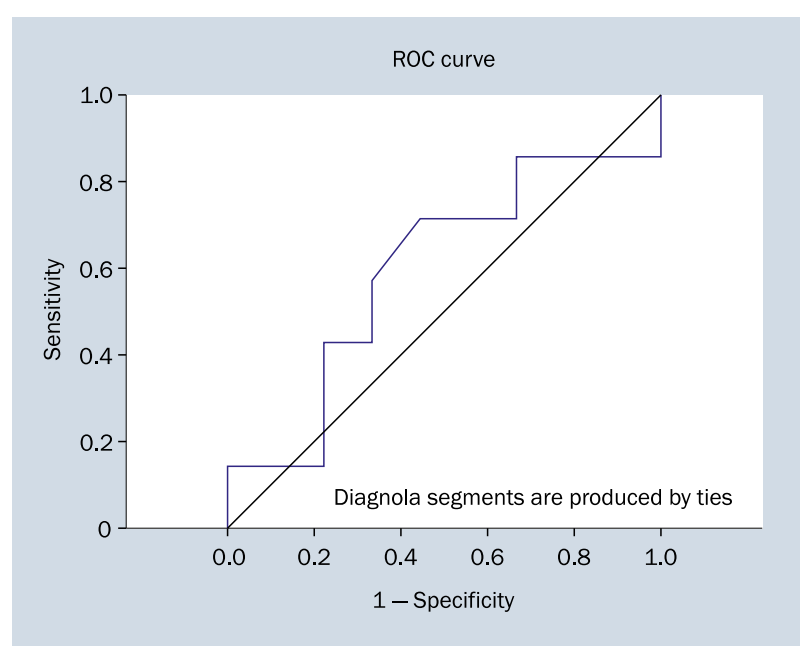

Figure 3. Receiver operating characteristic (ROC) analysis of intratympanic pressure in rest as predictor of barofunction result in group with unilateral poor Eustachian tube function-test; area under the curve $=0.595$, standard error $=0.151$

Since the barofunction test is measured in a nominal scale the application of correlation analysis is conditional. This is why we performed a ROC analysis, which is more suitable for bivariate data and provides information about the accuracy of a test (Figs. 2, 3).

The measure area under the curve (AUC) defines the accuracy of a given test. AUC values vary from 0 to 1 . A model whose predictions are $100 \%$ correct has an AUC of 1.0. A model whose predictions are $50 \%$ correct has an AUC of 0.5 . Values around 0.5 mean that a test is inaccurate, while values close to 1.0 are interpreted as a highly accurate test.

So far the results showed that the intratympanic pressure cannot be used as an accurate screening test for
Table 5. Correlation between the Eustachian tube function (ETF) test result and the barofunction test results for all divers

\begin{tabular}{llll}
\hline \multicolumn{2}{l}{ Correlations } & ETF result & Difficulties \\
\hline ETF & Pearson correlation & 1 & $-0.344^{*}$ \\
result & Sig. (2-tailed) & & 0.004 \\
& $N$ & 70 & 70
\end{tabular}

${ }^{*}$ Correlation is significant at the 0.01 level (2-tailed)

Table 6. Correlation between the Eustachian tube function (ETF) test result and the barofunction test results for divers with unilateral difficulties

\begin{tabular}{llll}
\hline \multicolumn{2}{l}{ Correlations } & ETF & Difficulties \\
\hline ETF & Pearson correlation & 1 & $-0.524 *$ \\
& Sig. (2-tailed) & & 0.037 \\
N & 16 & 16 \\
\end{tabular}

barofunction result neither in the whole sample (AUC $=0.592$, standard error $=0.112$ ), nor in the group with unilateral difficulties (AUC $=0.595$, standard error $=0.15$ ). Intratympanic pressure at rest is a static measure. In order to evaluate the diving fitness and Eustachian tube passability, a dynamic measure like the ETF test would be more suitable and informative.

This is why we compared the ETF test results with the BFT results. We discovered significant negative correlation between the ETF and BFT test results both in the whole sample $(r=-0.344, p<0.01)$ and in the group of divers with unilateral difficulties $(r=-0.524, p<0.05)$, meaning that higher ETF test results are related to lower risk of middle ear barotraumas (Tables 5 and 6).

In order to estimate the accuracy of the ETF test as a predictor for BFT result we conducted a ROC analysis (Fig. 4).

Unlike the results from tympanometry, we found that the ETF result is a highly accurate screening test for a successful BFT. This is why we compared the sensitivity and specificity at different values and confirmed that a value of $20 \mathrm{daPa}$ is the cut-off point for Eustachian tube passability sufficient for diving activities (Table 7).

In the separate group of divers with unilateral poor ETF test the graph is similar (Fig. 5, Table 8).

In the current study our results display very high accuracy of the ETF test as a screening tool of Eustachian tube passability (AUC $=0.984$, standard error $=0.025$ ). Considering a cut-off point of $20 \mathrm{daPa}$ (Table 8 ) we see that at this value the sensitivity of the ETF test is 0.889 and the specificity is 1.000 . Thus $88.9 \%$ of the cases with lower results than 20 daPa will have some Eustachian tube dysfunction, and $100 \%$ of the cases with a result higher than 20 daPa will have Eustachian tube passability, sufficient 


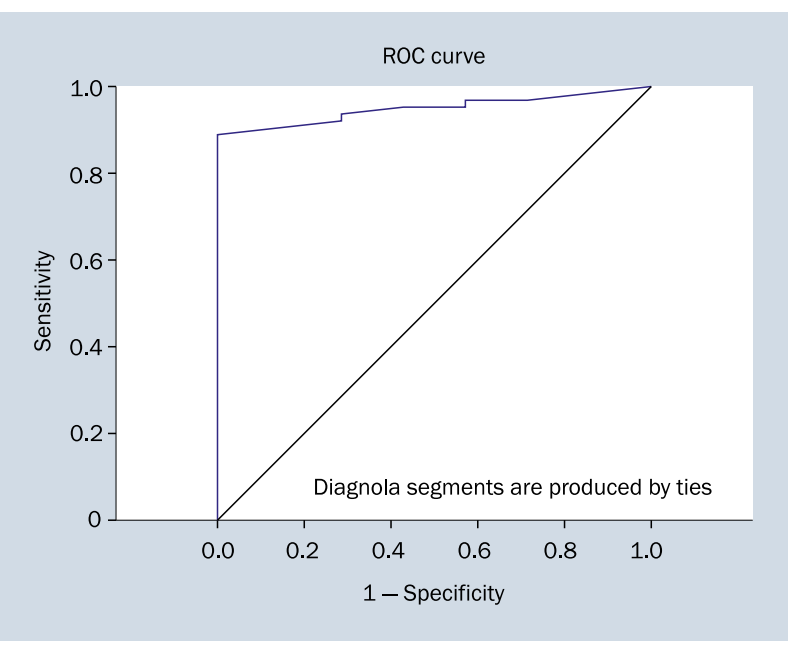

Figure 4. Receiver operating characteristic (ROC) curve of all Eustachian tube function test as predictor of barofunction result; area under the curve $=0.949$, standard error $=0.026$ : Eustachian tube function test can be used as a predictor of the barofunction test result

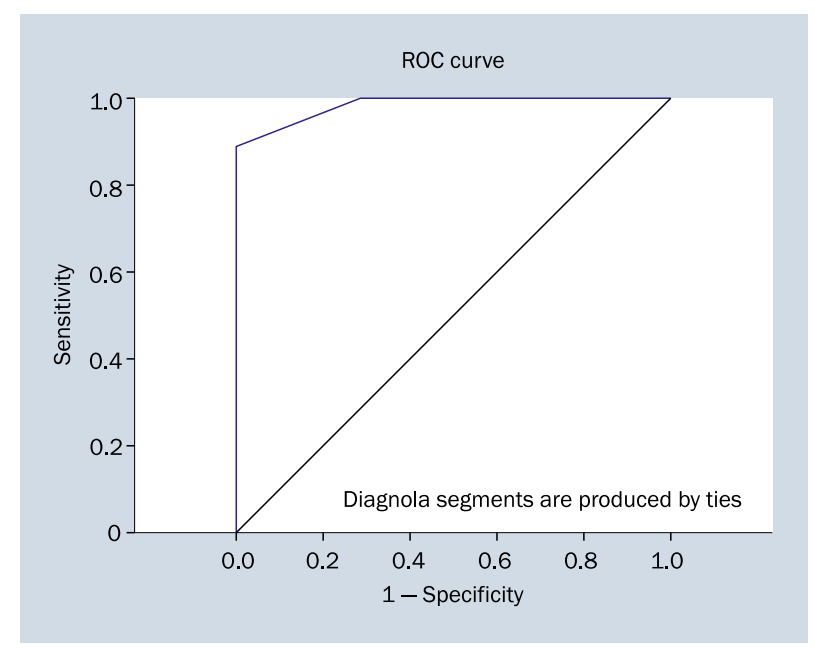

Figure 5. Receiver operating characteristic (ROC) analysis of unilateral poor Eustachian tube function test as predictor of barofunction result; area under the curve $=0.984$, standard error 0.025 . Eustachian tube function test can be used as a predictor of the barofunction test result

Table 7. Cut-off points of Eustachian tube function (ETF) test of all divers

$\begin{array}{lllllllllll}\text { ETF [daPa] } & 7 & 11.5 & 15.5 & 19.5 & 23 & 31.5 & 51 & 102 & 222 \\ \text { Sensitivity } & 1.000 & 0.968 & 0.952 & 0.889 & 0.841 & 0.746 & 0.635 & 0.302 & 0.016 \\ \text { Specificity } & 0.000 & 0.286 & 0.429 & 1.000 & 1.000 & 1.000 & 1.000 & 1.000 & 1.000\end{array}$

Table 8. Cut-off points in the group with unilateral poor Eustachian tube function (ETF) test

$\begin{array}{lllllllllllll}\text { ETF [daPa] } & 7 & 9 & 11.5 & 15.5 & 17 & 20 & 23 & 38.5 & 51 & 112 & 167 & 211 \\ \text { Sensitivity } & 1.000 & 1.000 & 1.000 & 1.000 & 1.000 & 0.889 & 0.778 & 0.556 & 0.444 & 0.222 & 0.111 & 0.000 \\ \text { Specificity } & 0.000 & 0.143 & 0.286 & 0.571 & 0.714 & 1.000 & 1.000 & 1.000 & 1.000 & 1.000 & 1.000 & 1.000\end{array}$

to prevent barotraumas. Based on these results we suggest using the ETF test with a cut-off point of 20 daPa as a simple, time-consuming screening tool for the success of a BFT and/or hyperbaric chamber examination and therapy.

\section{DISCUSSION}

In a previous study we discovered that values of the ETF test higher than 20 daPa guarantee normal Eustachian passability both for divers and people without diving experience. In cases of unilateral poor results of the ETF test and the barofunction, the main reason is not a flawed technique of execution of the test, but rather a real impassability of the Eustachian tube [6]. This can explain why in a study of divers who have already suffered barotraumas, Kitajima et al. [9] found more severe symptoms in unilateral accidents. The divers in the current study are highly qualified and they made no mistakes when performing the Valsalva manoeuvre. This excluded the influence of the factor incorrect technique of pressure equalisation, so curves of Figure 4 and Figure 5 are similar.

The results from the current study show that it is not necessary for a person with bilateral poor ETF test results to have difficulties in the BFT. These may be due to increased compliance of tympanal membrane of divers, careless execution of the ETF-test or using different pressure equalisation techniques in HBC (such as Frenzel manoeuvre, Lowry technique, Edmonds technique, etc.). Nevertheless, we can expect that if the person's ETF result is above $20 \mathrm{daPa}$, he/she would not suffer from a middle ear barotrauma because the Eustachian tube function is sufficient.

Taylor et al. [10] identified 16 studies involving seven different types of ETF tests and made conclusion that currently, no single test can be recommended to be used in the clinical practice. A combination of the 
nine-step test with other objective tests or patient-reported measures appears most promising as a core set of outcome measures for baro-induced Eustachian tube dysfunction.

Nine-step inflation/deflation test has a high accuracy as a predictor of middle ear barotrauma [11]. The test evaluates not only Eustachian passability, but functional state of pharyngeal structures. This way it is useful both on compression and decompression. Nevertheless, it is time consuming and complicated to implement. Most of middle ear barotrauma incidents with divers happen in time of compression. This is why we suggest that a simple test, measuring the effect of the Valsalva manoeuvre, is sufficient to predict barotraumas of the middle ear.

All divers with difficulty in the BFT were consulted with an otorhinolaryngology specialist. The main cause of the problems was nasal congestion due to an acute nasal infection. After adequate treatment, they repeated their BFT successfully.

\section{CONCLUSIONS}

Based on the above results we can make the following conclusions. Subjects with values of ETF test more than $20 \mathrm{daPa}$ have adequate passability of the Eustachian tube. Values of $20 \mathrm{daPa}$ and less are not a definitive predictor of the failure of the BFT. Results can prove to be useful in the usual work of an otolaryngologist to evaluate Eustachian function in cases of unilateral disease of middle ear.

\section{REFERENCES}

1. Taylor D. The Valsalva Manoeuvre: A critical review. South Pacific Underwater Medicine Society J. 1996; 26(1).

2. Kay E. Prevention of middle Ear Barotrauma. http://www.divingdoc. com/MEbaro.html.

3. Doyle WJ, Swarts JD, Banks J, et al. Sensitivity and specificity of Eustachian tube function tests in adults. JAMA Otolaryngol Head Neck Surg. 2013; 139(7): 719-727, doi: 10.1001/jamaoto.2013.3559, indexed in Pubmed: 23868429.

4. Beck DL, Spiedel DP, Prairie E, et al. Tympanometry and Acoustic Reflex: Innovation, Reviewand Application, Hearing Review, 3 April 2009.

5. Shupak A, Sharoni Z, Ostfeld E, et al. Pressure chamber tympanometry in diving candidates. Ann Otol Rhinol Laryngol. 1991; 100(8): 658-660, doi: 10.1177/000348949110000811, indexed in Pubmed: 1872517.

6. Georgiev K. Unilateral Eustachian Impasability - cutoff points for ETF test. Laryngorhinootologie. 2020; 99(S02): S314, doi: 10.1055/s0040-1711272.

7. http://mlwiki.org/index.php/ROC_Analysis.

8. Shopov N, Georgiev K, Vazharov Iv, Bozov Hr. Evaluation of Eustachian tubes function in divers. Congress paper 23 congress BMMR, TurkeyAntalya 11-14 May 2018.

9. Kitajima N, Sugita-Kitajima A, Kitajima S. [A study of the Eustachian tube function in patients with a scuba diving accident]. Nihon Jibiinkoka Gakkai Kaiho. 2012; 115(12): 1029-1036, doi: 10.3950/ jibiinkoka.115.1029, indexed in Pubmed: 23402207.

10. Tailor BV, Smith ME, Hutchinson PJA, et al. Outcome measures for baro-challenge-induced Eustachian tube dysfunction: a systematic review. Otol Neurotol. 2018; 39(2): 138-149, doi: 10.1097/ MA0.0000000000001666, indexed in Pubmed: 29315176.

11. Uzun C, Adali MK, Tas A, et al. Use of the nine-step inflation/ /deflation test as a predictor of middle ear barotrauma in sports scuba divers. Br J Audiol. 2000; 34(3): 153-163, doi: 10.3109/03005364000000125, indexed in Pubmed: 10905449. 